\title{
Training Motor Sequences: Effects of Speed and Accuracy Instructions
}

\author{
Jonathan S. Barnhoorn, Stefan Panzer, Ben Godde \& Willem B. Verwey
}

To cite this article: Jonathan S. Barnhoorn, Stefan Panzer, Ben Godde \& Willem B. Verwey (2018): Training Motor Sequences: Effects of Speed and Accuracy Instructions, Journal of Motor Behavior, DOI: 10.1080/00222895.2018.1528202

To link to this article: https://doi.org/10.1080/00222895.2018.1528202

\section{(c) 2018 The Author(s). Published with license by Taylor \& Francis Group, LLC}

曲 Published online: 05 Nov 2018.

Submit your article to this journal 중

Џلll Article views: 129

View Crossmark data $\asymp$

Citing articles: 1 View citing articles $₫$ 


\title{
Training Motor Sequences: Effects of Speed and Accuracy Instructions
}

\author{
Jonathan S. Barnhoorn ${ }^{1}$, Stefan Panzer ${ }^{2,4}$, Ben Godde ${ }^{3}$, Willem B. Verwey ${ }^{1,4}$ \\ ${ }^{1}$ Department of Cognitive Psychology \& Ergonomics, University of Twente, Enschede, The Netherlands. ${ }^{2}$ Institute of \\ Sport Science, Saarland University, Saarbrücken, Germany. ${ }^{3}$ Jacobs Center on Lifelong Learning and Institutional \\ Development, Jacobs University Bremen, Bremen, Germany. ${ }^{4}$ Department of Health \& Kinesiology, Texas A\&M \\ University, College Station, TX, USA
}

\begin{abstract}
Participants practiced a fixed 3- and a fixed 6-key press sequence for 144 times each. In the speed group, they were instructed to execute their sequences fast without bothering much about errors while the accurate group was instructed to be careful and prevent errors. In the test phase, participants executed series of 3 and 6 responses (a) when all element-specific stimuli were displayed in the familiar order, (b) in response to just the familiar first stimulus, and (c) by responding to random stimuli. The speed instruction yielded stronger sequencing skill while the accuracy instruction developed stronger reaction skill.
\end{abstract}

Keywords: training instruction, discrete sequence production task, motor skill, training

\section{Introduction}

\section{Different Sequence Representations}

A cquiring the skill to perform fixed movement patterns is generally assumed to involve the development of various sequence representations in memory (e.g., Hikosaka et al., 1999; Keele, Ivry, Mayr, Hazeltine, \& Heuer, 2003; Shea, Panzer, \& Kennedy, 2016; Verwey, Shea, \& Wright, 2015). Indications for this notion have been found in various sequencing study traditions such as the serial RT task (Abrahamse, Jiménez, Verwey, \& Clegg, 2010; Keele et al., 2003), the flexion-extension task (Shea \& Kovacs, 2013; Shea, Kovacs, \& Panzer, 2011), the finger-to-thumb opposition task (Karni et al., 1995), the $\mathrm{N} \times \mathrm{M}$ task (Hikosaka, Miyashita, Miyachi, Sakai, \& Lu, 1998), and the discrete sequence production task (DSP; Abrahamse, Ruitenberg, De Kleine, \& Verwey, 2013; Verwey, 1999). A recent review by Verwey et al. (2015) led to the conclusion that movement sequencing skill can be based on motor chunks at the motor processing level, verbal, and spatial (i.e., central-symbolic) representations at the central processing level (e.g., Barnhoorn, Döhring, Van Asseldonk, \& Verwey, 2016; De Lillo, Kirby, \& Poole, 2016; Verwey, Groen, \& Wright, 2016), and associations between representations of successive sequence elements at the various levels of information processing (cf. Abrahamse et al., 2010; Hunt \& Aslin, 2001). The contribution of each of these representations to the actual execution of the movement sequence is assumed to differ as a function of practice, task properties and individual differences (Hikosaka, Nakamura, Sakai, \& Nakahara, 2002; Shea et al., 2011). In the present study, we examined the possibility, suggested by two recent models of sequence execution, that the instruction to be accurate reduces the development of motor sequence representations relative to the instruction to be fast.

According to the Cognitive framework for Sequential Motor Behavior (C-SMB), central-symbolic representations develop already after, say, tens of trials (Verwey et al., 2015). These representations allow moderately fast execution of movement sequences but each movement still needs to be determined using an attention-demanding and time-consuming extraction of movement parameters from these central-symbolic representations. This happens when, for example, one enters the individual numbers of a familiar PIN or phone number on a keyboard with an unfamiliar layout. After hundreds of trials movement sequences gradually become coded by motor chunk representations. The motoric nature of these representations makes execution fast because the movement features can be retrieved directly from memory (cf. Hikosaka et al., 2002). Yet, this implies also that motor chunks are specific with respect to physical task features like force and effector used (Verwey \& Wright, 2004). Behavioral and neurophysiological studies suggest that these motor chunks are based on representations of successive hand postures rather than, for example, successions of finger movements (Graziano, Taylor, \& Moore, 2002; Rosenbaum, 2017; Rosenbaum, Loukopoulos, Meulenbroek, Vaughan, \& Engelbrecht, 1995). Support for different development rates of central-symbolic and motoric sequence representations comes for instance from indications that practice first induces effector-

Correspondence address: Willem B. Verwey, Department of Cognitive Psychology \& Ergonomics, University of Twente, P.O. Box 217, 7500 AE Enschede, The Netherlands. E-mail: w.b.verwey@utwente.nl

Jonathan Barnhoorn is now at TNO, Soesterberg, The Netherlands

This is an Open Access article distributed under the terms of the Creative Commons Attribution-NonCommercial-NoDerivatives License (http://creativecommons.org/licenses/by-nc-nd/4.0/), which permits non-commercial re-use, distribution, and reproduction in any medium, provided the original work is properly cited, and is not altered, transformed, or built upon in any way. 
unspecific accuracy - suggesting reliance on centralsymbolic representations - and only then an effector-specific speed increase - suggesting reliance on motor chunks (Hikosaka et al., 2002).

\section{Racing Processors}

According to the Dual Processor Model (DPM) the representations of up to about five individual movements can be loaded into a temporary motor buffer by a central processor (Abrahamse, Ruitenberg, De Kleine, \& Verwey, 2013; Verwey, 2001). This may involve retrieving a motor chunk representation from long-term memory in a single processing step, but also selecting and loading individual movements one by one into the motor buffer on the basis of element-specific stimuli or a central-symbolic representation (Sternberg, Monsell, Knoll, \& Wright, 1978; Verwey, 1996, 1999; Verwey, Abrahamse, De Kleine, \& Ruitenberg, 2014). Once the motor buffer has been loaded it is scanned by a motor processor that reads and executes each ensuing movement. While the motor processor is executing the motor buffer content, the central processor may be devoted to entirely different tasks (like counting tones, Verwey, Abrahamse, \& De Kleine, 2010; Verwey et al., 2014). However, it may be engaged also in selecting and preparing oncoming motor chunks (Kandel \& Perret, 2015; Verwey, 1995, 2001), and in translating key-specific stimuli into individual responses (Verwey, 1999; Verwey et al., 2014). In the latter case, the central processor, translating individual stimuli into responses, is racing with the motor processor to trigger each next movement (Verwey, 2001, 2003). The present study is aimed at exploring whether training instructions may influence the contributions of the various motor sequence representations and of these two processors.

\section{Execution Rate}

The mechanism generally assumed to be responsible for the various motor sequence representations consists of associations forming between the representations of individual movements at the verbal, spatial and/or motoric processing level. This associative learning mechanism has been proposed many years ago to underlie motor sequences like speech (e.g., Wickelgren, 1969), and has also been proposed to be involved in response selection skill (Hawkins, MacKay, Holley, Friedin, \& Cohen, 1973; Hommel, 2000; Logan, 1988; Pashler \& Baylis, 1991; Theios, 1975). In line with the old postulate that "neurons wire together if they fire together" (Hebb, 1949; Lowel \& Singer, 1992), new representations would develop when their constituents are simultaneously active and become linked. This associative mechanism has been argued to allow the neural system to learn (statistical and implicit) sequences in the serial
RT task (Abrahamse et al., 2010; Frensch \& Miner, 1994; Hunt \& Aslin, 2001), and also in the discrete motor sequences (Verwey et al., 2015). We assume associations to underlie both the development of motor chunks and central-symbolic representations.

Given the general assumption that activation of memory traces decays over time (Cowan, 1995; Frensch \& Miner, 1994; Hommel, 1994; McLean \& Shulman, 1978), the element association hypothesis implies that the development of associations reduces as the time between successive movement elements gets longer. Another effect of longer inter-movement intervals may be that other processes can occur during sequence execution that hinder association development, like the processing of response feedback. These seem two reasons that long inter-movement intervals hamper co-activation and therewith the development of sequence representations. A recent study explored this possibility and confirmed that sequence learning is reduced when response stimulus intervals (RSIs) are relatively long (Verwey \& Dronkers, 2018).

\section{The Present Study}

In the present study, we tested whether motor sequence learning is influenced by a speed instruction during practice. This issue is of practical and theoretical interest. Our reasoning was that a strategy to prevent errors would involve a longer-lasting reliance on stimuli in the reaction mode and therewith slow sequence execution. The longer time between successive responses could then hamper the development of sequence representations because of both longer inter-movement intervals and the occurrence of other processes in between successive sequence elements. Stimulus-response associations would however still develop. Instead a speed-oriented strategy could benefit the development of sequence representations due to a stronger co-activation of the successive (response) representations while the limited reliance on stimuli might reduce the development of stimulus-response associations at the response selection level. We used two sequences differing in length to see whether the anticipated effects occur irrespective of sequence length.

To examine the effect of instructions emphasizing either speed or accuracy, we instructed participants of the so-called speed group to practice a 3-key and a 6key discrete key pressing sequence at a high rate while bothering little about errors. Participants of the accurate group practiced the same sequences, but they were told to be careful and prevent errors as much as possible. In the ensuing test phase, we examined whether motor sequencing skill was different for these two groups ${ }^{1}$. The test phase was identical for the two groups and included three conditions. The random condition involved 3- and 6-element sequences that differed on each trial. This 
condition was included to determine whether the instruction would strengthen response selection skill. This response selection skill may be better developed in the accurate than the speed group, but given the high stimulus-response compatibility large benefits were not expected. In the single-stimulus test condition participants executed the entire 3- and 6-key sequences they had been practicing in response to just the first stimulus of each sequence (cf. Verwey, 1999; Verwey, Abrahamse, Ruitenberg, Jiménez, \& De Kleine, 2011). If the speed instruction had indeed stimulated the development of central-symbolic and motor chunk sequence representations, execution in the single-stimulus condition should be faster and more accurate for the speed than for the accurate group. Finally, the familiar condition of the test phase allowed participants to rely on sequence representations while also using key-specific stimuli - just like during practice. In earlier studies it has been argued that the execution of familiar sequences in which all key-specific stimuli are displayed, is based primarily on sequence representations but that key-specific stimuli are still used as well (Abrahamse et al., 2013; Verwey, 2001, 2003). At the outset it was not clear whether a greater reliance on key-specific stimuli in the accurate group could compensate for the expected stronger sequence representations in the speed group.

\section{Method}

\section{Participants}

Twenty-four participants (aged 19-28, mean age 23.2, 17 females) took part in the experiment. They were randomly allocated to the accurate group (aged 20-28, mean age 23.0, 8 females) and the speed group (aged 19-28, mean age 23.4, 9 females). Most of them were students at the University of Twente. The ethics committee of the University of Twente, Faculty of Behavioral, Management and Social Sciences, approved the study. All participants provided written informed consent.

\section{Apparatus}

The task was carried out on two 15-inch laptops running E-Prime 2.0 (Asus pro55s, Samsung R530). The use of these laptops was counterbalanced across the speed and accurate groups. All unnecessary services like virus scanners and updates were deactivated and the laptops were connected to a wall-outlet during the whole experiment. The same external standard USB keyboard was used with both laptops. Participants were tested in their living environment.

\section{The Discrete Sequence Production Task}

Participants practiced a 3- and a 6-key sequence, displayed in pseudorandom order, using the ring, middle and index fingers of both hands. They used the D, F, G, $\mathrm{J}, \mathrm{K}$, and $\mathrm{L}$ keys of a standard computer keyboard to respond to targets presented in one of six horizontally aligned $25 \times 25 \mathrm{~mm}$ placeholders. After pressing the spatially compatible key, the next target was immediately displayed (i.e., RSI $=0$ ). This continued until a sequence was completed. We use the term trial to denote performance of one sequence. After each trial, a $2 \mathrm{~s}$ white screen was presented, the next trial then started with a $1000 \mathrm{~ms}$ presentation of empty placeholders. When a key was pressed during this presentation period, an error message "too early" was presented for $1500 \mathrm{~ms}$ and the presentation period was restarted. To ensure that across participants all fingers were used equally often at each sequential position, the sequence elements were rotated across sequential positions (e.g., the sequence $\mathrm{G}$, $\mathrm{L}, \mathrm{D}$ for one participant becomes $\mathrm{J}, \mathrm{D}, \mathrm{F}$ for the next, and so on). As a reminder of the keys to be used, the letter $\mathrm{H}$ was displayed in between Placeholders 3 and 4 to emphasize that the three keys left and right of the $\mathrm{H}$ key were associated with the placeholders. The horizontal spacing was $51 \mathrm{~mm}$ between the third and fourth placeholder, and $13 \mathrm{~mm}$ between the other placeholders. The screen background was white and the placeholder outlines were black. A placeholder was filled green when it became the active target.

The DSP task involved 6 practice blocks each containing 2 sub-blocks of 24 trials each (48 trials per block, 144 practice trials per sequence). Although there was no specific familiarization phase, the experimenter monitored performance of the first few sequences to ensure that the participant understood the task. Participants were given a 40-second break between sub-blocks and a 120second break between blocks. The instructions and feedback during practice urged the participants to produce the sequences either fast or accurately.

During the breaks, the speed group received information about their mean RT after each completed subblock. When the mean RT was $2.5 \%$ faster than the previous block, the instruction read (in Dutch): "This was faster than the previous block, good job! Try to be faster again in the next block". When RT improvement did not meet the $2.5 \%$ criterion, the instruction read: "Unfortunately you didn't improve yourself sufficiently! Try to be faster in the next block". When error-rates, calculated as the percentage of trials containing an error, were below $10 \%$, participants were urged to be faster with the instruction "You are allowed to make more errors". Only when error-rates were above $40 \%$ the instruction was "You are making too many errors, please try to make fewer errors".

In the accurate group, participants were stimulated to perform the task without making errors. The message during the breaks was either "You did not make any errors during the previous block, good job! Please try to 
maintain this", or "You made X errors during the previous block, try to make fewer errors". When a participant's mean key-press RT across a sub-block surpassed $2000 \mathrm{~ms}$, the only instruction was "You responded a little too slowly, try to respond faster". After committing an error, the message "wrong" was presented with both groups. To penalize errors to a stronger extent in the accurate group, the error feedback duration was $3000 \mathrm{~ms}$ in the accurate group, and $1500 \mathrm{~ms}$ in the speed group. Even though this was never explicitly tested, the resulting slower progress in the accurate group was expected to annoy participants in that group and motivate them even more to prevent errors.

The test phase consisted of three sub-blocks, each containing 32 trials (16 per sequence) and involving a different experimental condition. No instructions were given as to the speed-accuracy tradeoff and participants were assumed to no longer adhere to the earlier instructions. Each false key press was immediately followed by a breaking off of the sequence and an error message. The three sub-blocks were separated by a 40 -second break. The first two sub-blocks included the familiar and the random conditions in an order counterbalanced across participants. In the familiar condition, the sequences were carried out as during practice (but without speed or accuracy instruction), and the order of the 3- and 6-key sequences was again pseudorandom. The random condition consisted of sequences with a random order of elements that was determined each time anew for each sequence (though an element was never immediately repeated).

The single-stimulus block always followed the two other test conditions and involved the two familiar sequences too but, after responding correctly to the first stimulus, no key-specific stimuli were displayed anymore. The participants were instructed to complete the sequence by heart (the two sequences a participant had practiced always started with different sequence elements). Participants were informed on the screen before the single-stimulus condition that stimuli after the first would not be displayed. They started after confirming to have understood the instructions.

\section{Procedure}

Participants were randomly assigned to either the speed or the accurate group. Before each of the 6 practice blocks the instructions were provided to stimulate participants to perform either fast or accurately. After the practice phase, an awareness questionnaire was administered. It asked participants to write down the sequences using the letters of the keys they had been pressing. To remind the participants of the letters on each of the keys, the questionnaire contained a picture of the keys and their letters. The session was completed by the test phase consisting of three sub-blocks, each containing another condition.

\section{Analyses}

We excluded all trials that had a mean RT exceeding a threshold of the mean trial RT plus 2.5 times the standard deviation of mean RTs in that sub-block. For accuracy analyses we calculated across all trials in each block the proportions of trials containing an error. We then performed an arcsine transformation on the proportions of erroneous trials before submitting the resulting variable to the ANOVA (Winer, Brown, \& Michels, 1991). When assumptions of sphericity were violated for any of the analyses we applied the Greenhouse-Geisser correction, we report the corrected $p$-values and original degrees of freedom. When reporting RTs, we use $T_{n}$ to denote the RT to the $n$ 'th stimulus. Awareness results and subjective strategies were analyzed by comparing the numbers of participants in each instruction group with and without awareness, and the use they indicated to have made of a particular reproduction strategy.

\section{Results}

\section{Practice Phase}

Response times of the 3-key sequence in the practice phase were submitted to a 2 (Group: speed vs. accurate $) \times 6 \quad($ Block $) \times 3 \quad$ (Key Position: 1 to 3$)$ mixed ANOVA with Group as between-subjects variable. As expected, participants in the speed group were considerably faster than participants in the accurate group (260 vs. $360 \mathrm{~ms}), \quad F(1,22)=12.0, \quad p=.002, \quad \eta_{p}{ }^{2}=.35 \quad$ (see Figure 1). Reaction times generally reduced over successive blocks, $F(5,110)=103.3, p<.001, \eta_{p}{ }^{2}=.82$, and differed across keys, $F(2,44)=125.4, p<.001, \eta_{p}{ }^{2}=.85$. Key Position interacted with Block, $F(10,220)=23.0$, $p<.001, \eta_{p}{ }^{2}=.51$, indicating that $\mathrm{T}_{2}$ and $\mathrm{T}_{3}$ reduced more with practice than $\mathrm{T}_{1}$. The Key Position $\times$ Group interaction, $F(2,44)=10.3, p<.001, \eta_{p}{ }^{2}=.32$, showed for the speed group the typical phenomenon in DSP sequences that $T_{2}$ and $T_{3}$ are about as fast $\left(T_{1}-T_{3}\right.$ : $417 \mathrm{~ms}, 184 \mathrm{~ms}, 178 \mathrm{~ms}$, respectively), while this was less so for the accurate group $\left(\mathrm{T}_{1}-\mathrm{T}_{3}: 459 \mathrm{~ms}, 355 \mathrm{~ms}\right.$, $267 \mathrm{~ms}$, respectively). The Block $\times$ Group interaction and the three-way interactions were not statistically significant $(p s>.30)$.

Response times of the 6-key sequence were submitted to a 2 (Group) $\times 6$ (Block) $\times 6$ (Key Position) mixed ANOVA. Again, participants in the speed group were faster than participants in the accurate group (227 vs. $346 \mathrm{~ms}), \quad F(1,22)=13.6, p=.001, \eta_{p}{ }^{2}=.38$, and performance of the 6-key sequence improved over blocks, $F(5,110)=119.0, p<.001, \eta_{p}{ }^{2}=.84$ (Figure 1). The main effect of Key Position was significant, $F(5,110)=35.3, p<.001, \eta_{p}{ }^{2}=.62$, as was the Key Position $\times$ Block interaction, $F(25,550)=10.8, p<.001$, $\eta_{p}{ }^{2}=.33$. Contrary to the 3 -key sequence analysis, the 


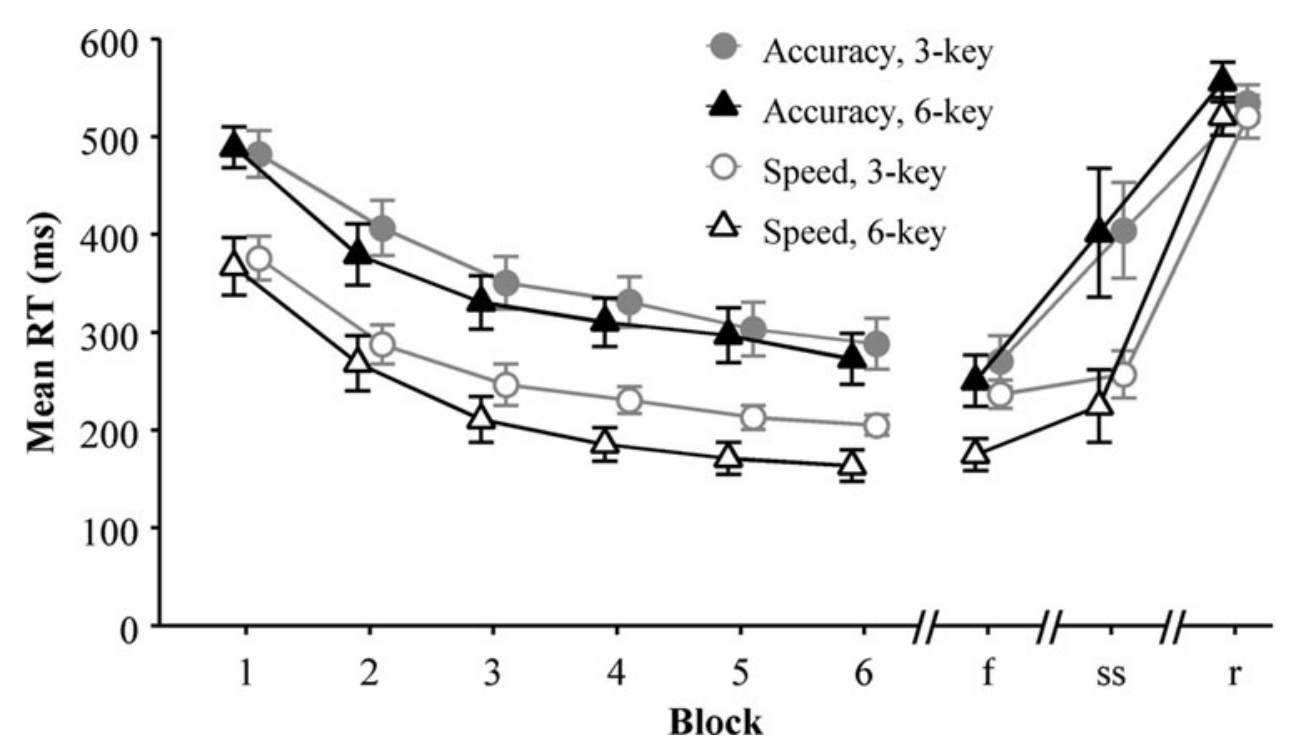

FIGURE 1. Mean RTs in practice Blocks 1 to 6 and the test conditions, $\mathrm{f}=$ familiar, ss $=$ single-stimulus ( $n=22$ for the 6-key sequence, $n=24$ for the 3-key sequence), $\mathrm{r}=$ random. The order of the familiar and random test conditions was counterbalanced across participants and was always followed by the single-stimulus condition. Error bars in this and the other figures indicate the standard error of the mean.

Key Position $\times$ Group interaction did not reach significance, $F(5,110)=1.88, p=.10$. The Block $\times$ Group and the three-way interactions were again not significant $(p \mathrm{~s}>.28)$.

For the 3- and 6-key sequences, arcsine transformed proportions of trials correct per block (see, Winer et al., 1991) were submitted to 2 (Group) $\times 6$ (Block) mixed ANOVAs. As intended participants in the speed group were less accurate than their colleagues in the accurate group in both the 3-key sequence $(92.7 \%$ vs. $98.4 \%)$, $F(1,22)=13.2, \quad p=.001, \quad \eta_{p}{ }^{2}=.38, \quad$ and the 6-key sequence $(76.1 \%$ vs. $95.8 \%), F(1,22)=32.4, p<.001$, $\eta_{p}{ }^{2}=.60$ (see Figure 2). The main effect of Block and the Block $\times$ Group interaction effect were not significant for the 3-key sequence. However, for the 6-key sequence, accuracy reduced with block, $F(5,110)=3.2$, $p=.009, \eta_{p}{ }^{2}=.13$. In fact, this effect was caused by the speed group, for which accuracy reduced (from $85.8 \%$ in Block 1 to $70.4 \%$ in Block 6), $F(5,110)=3.6, p=.005$, $\eta_{p}{ }^{2}=.14$, whereas accuracy remained quite stable in the accurate group (around 95\%).

\section{Test Phase}

Response times of the 3-key sequence in the test phase were submitted to a 2 (Group: speed vs. accurate) $\times 3$ (Test: familiar, random, single-stimulus) $\times 3$ (Key Position) mixed ANOVA with Group as between-subjects variable. In line with the absence of a particular speed-accuracy instruction for both groups in the test phase, the groups no longer differed significantly with respect to the 3-key sequence execution rate across the three test conditions, $F(1,22)=3.7, p=.07, \eta_{p}{ }^{2}=.14$. The Test main effect was significant, $F(2,44)=124.2$, $p<.001, \eta_{p}{ }^{2}=.85$, and, more importantly, the Test by Group interaction showed that the disadvantage of the accurate relative to the speed group was larger in the single stimulus $(147 \mathrm{~ms})$ than in the familiar and random conditions (34 and $14 \mathrm{~ms}$, respectively), $F(2,44)=8.1$, $p=.006, \eta_{p}{ }^{2}=.27$. Planned comparisons confirmed that the advantage of the speed over the accurate group was larger in the single-stimulus than in the familiar condition, $F(1,22)=11.0, p=.003, \eta_{p}{ }^{2}=.33$, and larger in the single-stimulus than in the random condition, $F(1,22)=7.9, p=.01, \eta_{p}{ }^{2}=.26$. In fact, the speed group was $147 \mathrm{~ms}$ faster than the accurate group in the singlestimulus condition, $F(1,22)=7.4, \quad p=.01, \quad \eta_{p}{ }^{2}=.25$, while there was no significant group difference for the familiar $(34 \mathrm{~ms})$ and the random $(30 \mathrm{~ms})$ conditions, $F_{\mathrm{s}}(1,22)<1.24, p \mathrm{~s}>.28$ (Figures 1 and 3 ). Finally, the effect of Key Position was significant, $F(2,44)=80.9$, $p<.001, \eta_{p}{ }^{2}=.79$, the Key Position $\times$ Group interaction was marginally significant, $F(2,44)=3.1, \quad p=.06$, $\eta_{p}{ }^{2}=.12$, and the Key Position $\times$ Test interaction was significant, $F(4,88)=29.6, p<.001, \eta_{p}{ }^{2}=.57$ (Figure 3).

Response times of the 6-key sequences in the test phase were analyzed with a 2 (Group) $\times 3$ (Test) $\times 6$ (Key Position) mixed ANOVA. The main effect of Group was significant, $F(1,20)=5.4, \quad p=.031$, $\eta_{p}{ }^{2}=.21^{2}$, as was the main effect of Test, $F(2,40)=74.6, \quad p<.001, \quad \eta_{p}{ }^{2}=.79$. The Group $\times$ Test 


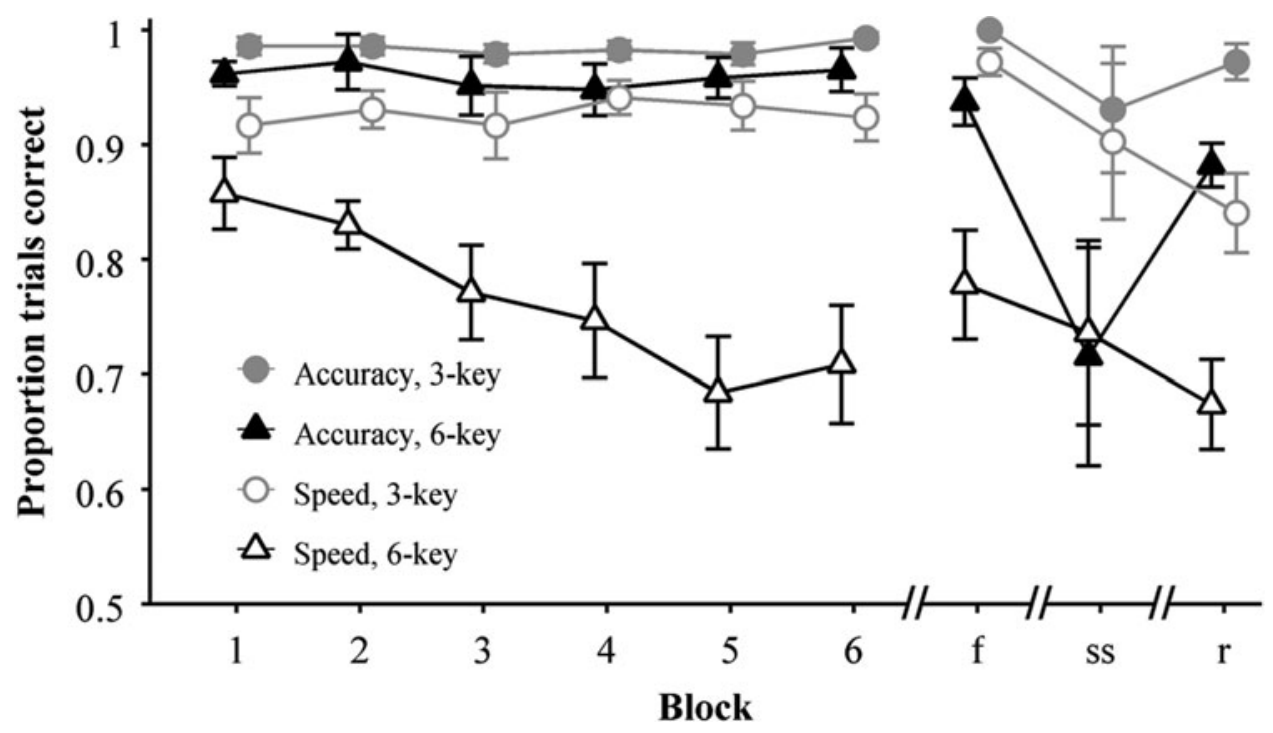

FIGURE 2. Proportion of correct trials in practice blocks 1 to 6 and the test conditions, $\mathrm{f}=$ familiar, ss $=$ single-stimulus ( $n=24$ for both sequences), $\mathrm{r}=$ random.

interaction indicated that the difference between the speed and accurate groups differed across the three Test conditions, $F(2,40)=3.8, p=.03, \eta_{p}{ }^{2}=.16$ (Figure 1). Planned comparisons confirmed our expectation for the 6-key sequences, too, that the accurate group was slowed more than the speed group in the single-stimulus condition. This was indicated by the advantage of the speed over the accurate group being the largest in the singlestimulus condition: Planned comparisons showed that the execution rate of the speed group was significantly higher in the single-stimulus than in the random condition, $F(1,20)=4.4, p=.05, \eta_{p}^{2}=.18$, and tended to be higher in the single-stimulus than in the familiar condition, $F(1,20)=3.4, p=.08, \eta_{p}^{2}=.34$. This relatively large advantage of the speed over the accurate group in the single-stimulus condition was corroborated by planned comparisons. These showed that in the singlestimulus condition the speed group was $177 \mathrm{~ms}$ faster than the accurate group, $F(1,20)=5.5, p=.03, \eta_{p}{ }^{2}=.22$. In contrast, the speed group was only a marginally significant $64 \mathrm{~ms}$ faster in the familiar condition, $F(1,20)=3.9, p=.06, \eta_{p}{ }^{2}=.16$, and not faster in the random condition (which difference amounted to $30 \mathrm{~ms}$ ), $F(1,20)=1.0, p=.33$. Finally, the main effect of Key Position was significant, $F(5,100)=40.5, \quad p<.001$, $\eta_{p}{ }^{2}=.67$, and Key Position interacted with Test, $F(10,200)=10.3, p<.001, \eta_{p}{ }^{2}=.34$. The other interactions did not reach significance.

Arcsine transformed proportions of correct sequences per block were submitted to two 2 (Group) $\times 3$ (Test) mixed ANOVAs, one for each sequence length (Figure 2 ). Speed participants generally produced fewer accurate sequences than accurate participants when performing the 3-key sequence $(90.5 \%$ vs. $97.0 \%$, respectively, Figure 2), $F(1,22)=6.76, p=.016, \eta_{p}{ }^{2}=.24$, and the 6key sequence $(73.7 \%$ vs. $84.6 \%$, respectively), $F(1,22)=4.10, p=.055, \eta_{p}{ }^{2}=.16$. So, across the test conditions the two groups appear to still have followed the practice phase instructions in the test phase. For the 3-key sequence, the effect of Test condition was significant, $F(2,44)=4.2, \quad p=.021, \eta_{p}{ }^{2}=.16$, and accuracy amounted to $98.6 \%, 90.7 \%$, and $92.2 \%$, for the familiar, random, and single-stimulus conditions, respectively. For the 6-key sequences, accuracy did not differ significantly across the three test conditions, $F(2,44)=2.3, p=.11$. The Group by Test interactions did not reach significance in either ANOVA ( $p s>.13)$, but given that our predictions concerned error rate too, we compared accuracy differences between the speed and accurate groups for each test condition with planned comparisons. This showed a higher accuracy for the accurate than for the speed group in the familiar sequences: The accurate group had 2.8\% more accurate 3-key sequences than the speed group, and $15.8 \%$ more accurate 6-key sequences, $F \mathrm{~s}(1,22)>5.4, p \mathrm{~s}<.03, \eta_{p}{ }^{2} \mathrm{~s}>.20$. In the random condition, the accurate group was more accurate than the speed group too (3-key: 12.9\% more accurate; 6-key: $19.6 \%$ more accurate sequences $), F \mathrm{~s}(1,22)>10.2, p \mathrm{~s}=$ $<.005, \eta_{p}{ }^{2} \mathrm{~s}>.32$. Only in the single-stimulus condition accuracies showed no group differences (advantages for the accurate group: $3.7 \%$ in the 3-key sequence, $-2.7 \%$ in the 6-key sequence), $F \mathrm{~s}(1,22)<0.57, p \mathrm{~s}>.45$.

In summary, the RT and accuracy analyses showed the same patterns of execution rates and accuracies for 

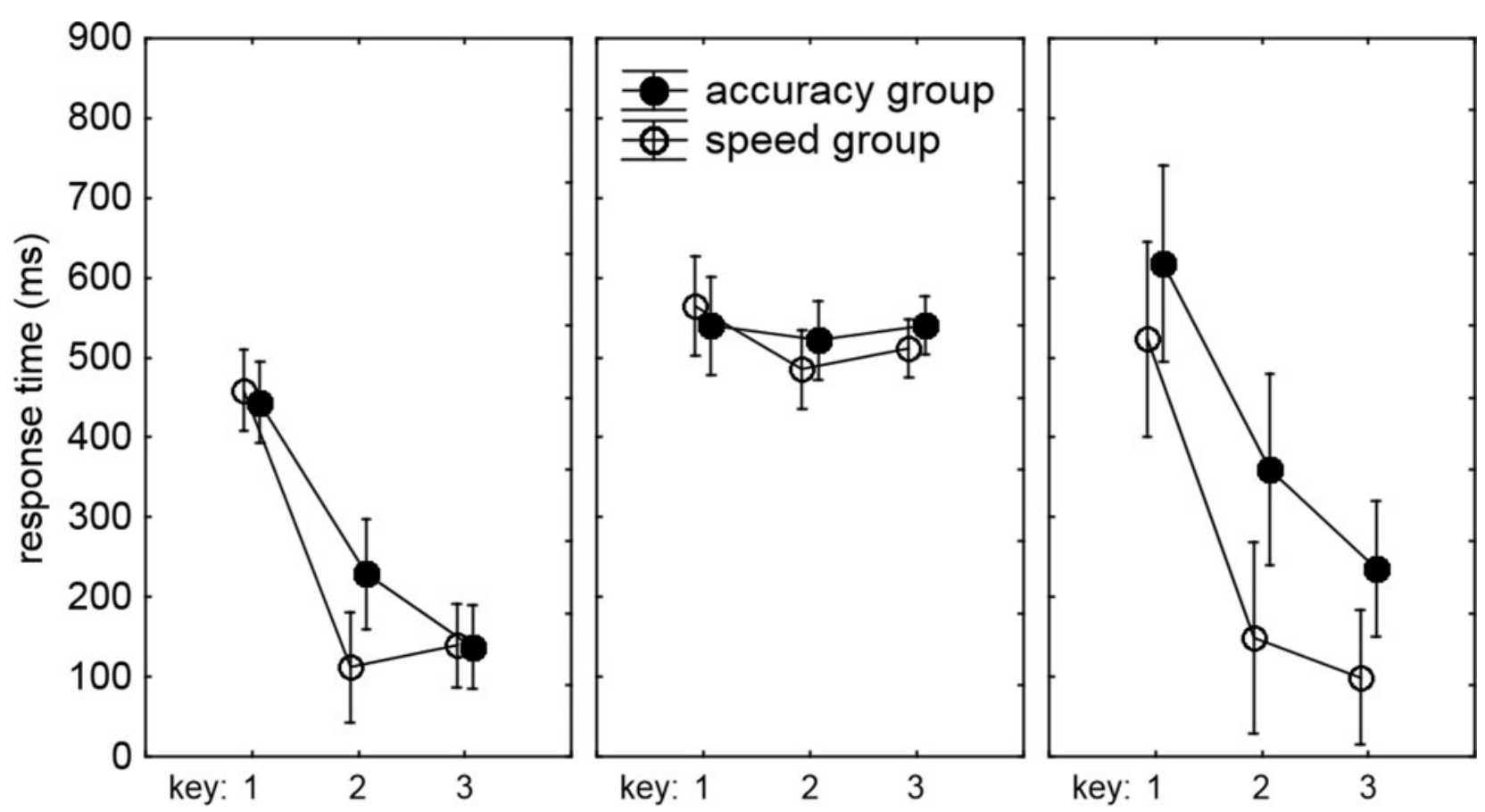

familiar

random

single-stimulus
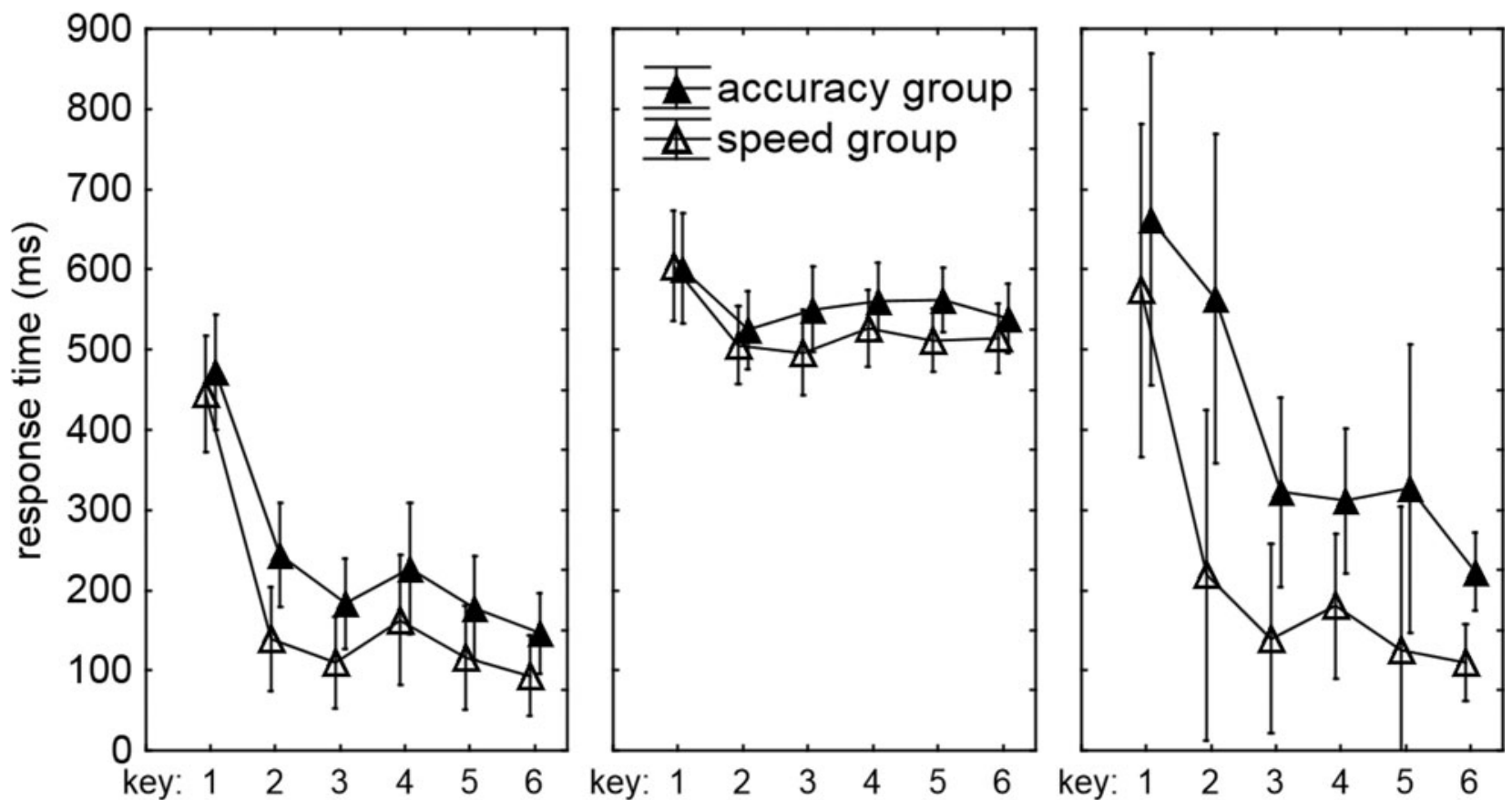

familiar

random

\section{single-stimulus}

FIGURE 3. RTs of the speed and accurate groups in the test phase, as a function of sequence length, test condition, and key position (the 6-key sequence data are based on 22 participants). 
3- and 6-key sequences in the test phase. The singlestimulus sequences were performed $147 \mathrm{~ms} / 177 \mathrm{~ms}$ faster (for 3-/6-key sequences, respectively) by the speed group than by the accurate group while their accuracies did not differ. In other words, the speed group was more skilled executing sequences without key-specific stimuli than the accurate group. In contrast, in both the familiar and random conditions execution rate was not different for the two groups, but the accurate group was more accurate. In the random condition accuracy of the accurate group was a significant 12.9\%/19.6\% higher (for 3-/6key sequences, respectively) than of the speed group, and in the familiar condition the accurate group advantage amounted to $2.8 \% / 15.8 \%$, respectively, which effects were statistically significant too.

\section{Awareness}

Awareness appeared quite high. Twenty-three of the 24 participants were able to write down their 3-key sequences, and 19 of these participants did so with their 6-key sequences. Only 1 participant could not write down either of her sequences. Practice instruction did not affect awareness in that 11 speed and 8 accurate participants had written down both sequences correctly (Fisher Exact test, $p=.32$ ). The correlations between the number of correctly written sequence elements and mean sequence execution rate for the 6-key sequences in block 6 were far from significant $(p s>.52)$. Five speed participants and 4 accurate participants indicated to have replayed, in their mind or on the table top, executing their sequences in order to fill in the awareness questionnaire. Of the remaining participants, 2 speed and 4 accurate participants indicated to have remembered the spatial order of the stimuli and/or keys. So, the speed and accurate instructions did not differently influence awareness of the sequences.

\section{Discussion}

The present study was designed to test predictions of the DPM and the C-SMB models. Their assumption that executing a familiar keying sequence involves a race between reacting to key-specific stimuli and reading the sequence representation (central-symbolic or motor chunk) in the motor buffer suggested that the instruction to be either fast or accurate would benefit more the development of either sequence representations or stimulus-response associations. The results confirm this prediction in that in the single-sequence condition, where execution relies solely on sequence representations, the practiced sequences were executed fastest by the speed group. Instead, the accurate group appeared to have become more accurate than the speed group when responding to the key-specific stimuli in the random and also in the familiar condition.

\section{Sequencing Skill}

Given the many indications that the execution of familiar keying sequences benefits from the continued display of key-specific stimuli (e.g., Ruitenberg, Verwey, Schutter, \& Abrahamse, 2014; Verwey, 1999; Verwey, 2001, 2003), we considered the single-stimulus test condition the best indicator for motor sequencing skill. The results confirm this in that the main difference between the speed and accurate groups was that the accurate group slowed considerably when key-specific stimuli were no longer displayed in the single-stimulus test condition while execution rate of the speed group hardly reduced. This speed difference in the single-stimulus condition cannot be attributed to different speed-accuracy tradeoffs in the two groups as accuracy was not different for the two groups. So, as predicted practicing with higher execution rates promoted the development of sequencing skill, and the higher error rate of the speed group during practice did not hamper the development of this sequencing skill. Importantly, the accurate group was able to execute the practiced sequences in the single-stimulus condition too. This shows that accurate participants did develop sequencing skill too. Given the similar awareness in both groups, that skill most likely involved central-symbolic sequence representations and not so much motor chunks ${ }^{3}$.

The similarity in awareness in both groups implies that the execution rate advantage of the speed group in the single-stimulus condition cannot be attributed to more extensive explicit sequence knowledge. Most likely, the processes developing explicit sequence knowledge in the DSP task - like testing hypotheses on element order (Rünger \& Frensch, 2008, Rünger \& Frensch, 2010) - do not rely on the interval between successive responses. Indeed, the DPM indicates that in the DSP task such central processes can initially occur in between successive DSP task sequences, and may concur with sequence execution once these sequences have been practiced extensively and are carried out by the motor processor (as was for example shown with a counting task, Verwey et al., 2014). In line with this reasoning longer RSIs during practice appeared not to increase awareness (Verwey \& Dronkers, 2018). So, while lower execution rates in the serial RT task are associated with higher awareness in the serial RT task (Cleeremans \& Sarrazin, 2007; Haider \& Frensch, 2009; Rünger \& Frensch, 2008, Rünger \& Frensch, 2010), this seems not the case in the DSP task with its long inter-sequence intervals and its reduced reliance on the central processor.

\section{Response Skill}

While we did not expect a large effect of the accuracy instruction on response selection skill because the stimulusresponse mappings were spatially compatible, the data did show that the accurate group performed more accurately 
when key-specific stimuli were presented in the random and the familiar conditions, while that was not the case in the single-stimulus condition. That practice strengthens compatible stimulus-response mappings is in line with dual route models of response selection skill that assume automation of response selection by the development of stimulus-response associations that prime the response after which rule- (or algorithm-) based mechanisms verify the activated response (Hommel, 2000; Kornblum, Hasbroucq, \& Osman, 1990; Logan, 1988; Pashler \& Baylis, 1991; Proctor \& Vu, 2013). The finding that the accurate participants were more accurate than the speed participants in the familiar condition, too, provides further support for the assumption of the DPM that in the typical familiar condition stimuli are still used, and that in that situation response selection and sequence execution are racing to trigger the individual movements of a familiar sequence (Abrahamse et al., 2013; Verwey et al., 2015).

\section{Conclusions}

The present data confirm the prediction of the DPM and the C-SMB that the instruction to be fast during practice benefits especially the development of sequence representations that control execution via response-response associations (like central-symbolic and motor chunk representations), while the instruction to be accurate during practice benefits especially the accuracy of selecting individual responses via stimulus-response associations. This group difference cannot be attributed to differences in awareness of the sequence. While we did not expect a strong effect of our instructions on selecting spatially-compatible responses there still was an accuracy benefit of the accurate group in the conditions in which key-stimuli were displayed, even when in the familiar condition sequence representations could be used too. That practice may strengthen even compatible stimulus-response mappings is in line with dual route models of response selection skill that assume automation of response selection by the development of stimulus-response associations. The finding that in the familiar condition accuracy benefitted when keyspecific stimuli were displayed supports the assumption that executing the individual movements in a familiar sequence involves a race between stimulus-based response selection and sequence execution skill.

\section{NOTES}

1. We here do not discuss the speed-accuracy tradeoff literature that is usually focused on choice RT tasks (e.g., Bogacz, Wagenmakers, Forstmann, \& Nieuwenhuis, 2010; Heitz, 2014; Wickelgren, 1977), as those studies usually involve neither learning nor executing movement sequences.
2. In both the speed and the accurate group one participant was excluded from RT analysis because he/she did not complete a single 6-key sequence in the single stimulus condition.

3. We also assessed the development of sequence learning with the Initiation-Execution Difference (IED), which is the difference between initiation of the sequence and the subsequent execution intervals while potential concatenation of successive motor chunks is excluded (Barnhoorn, Van Asseldonk, \& Verwey, in press). While IED previously indicated the expected faster sequence learning in younger than in older participants, the present IED analysis did not show a significant group difference in the test phase. It did show a larger IED in the singlestimulus than in the familiar condition which can be attributed to a longer preparation time in the single-stimulus condition.

\section{DISCLOSURE STATEMENT}

Author J. S. Barnhoorn is supported by the aforementioned grant and declares that he has no conflict of interest. Author S. Panzer declares that he has no conflict of interest. Author B. Godde declares that he has no conflict of interest. Author W. B. Verwey declares that he has no conflict of interest.

\section{FUNDING}

This study was funded by the Netherlands Organization for Scientific Research (NWO) under contract number 464-13-157 as part of the ORA reLoad project. This grant was awarded to W. B. Verwey and E. H. F. Van Asseldonk.

\section{ETHICAL STANDARDS}

All procedures performed in studies involving human participants were in accordance with the ethical standards of the institutional and/or national research committee and with the 1964 Helsinki declaration and its later amendments or comparable ethical standards. Informed consent was obtained from all individual participants in the present study.

\section{REFERENCES}

Abrahamse, E. L., Jiménez, L., Verwey, W. B., \& Clegg, B. A. (2010). Representing serial action and perception. Psychonomic Bulletin \& Review, 17(5), 603-623.

Abrahamse, E. L., Ruitenberg, M. F. L., De Kleine, E., \& Verwey, W. B. (2013). Control of automated behaviour: Insights from the discrete sequence production task. Frontiers in Human Neuroscience, 7(82), 1-16. 
Barnhoorn, J. S., Döhring, F. R., Va Asseldonk, E. H. F., \& Verwey, W. B. (2016). Similar representations of sequence knowledge in young and older adults: A study of effector independent transfer. Frontiers in Psychology, 7(1125), $1-10$.

Barnhoorn, J. S., Van Asseldonk, E. H. F., \& Verwey, W. B. (in press). Differences in chunking behavior between young and older adults diminish with extended practice. Psychological Research, 1-11.

Bogacz, R., Wagenmakers, E.-J., Forstmann, B. U., \& Nieuwenhuis, S. (2010). The neural basis of the speed-accuracy tradeoff. Trends in Neurosciences, 33(1), 10-16.

Cleeremans, A., \& Sarrazin, J. (2007). Time, action, and consciousness. Human Movement Science, 26(2), 180-202.

Cowan, N. (1995). Attention and memory: An integrated framework. New York: Oxford University Press.

De Lillo, C., Kirby, M., \& Poole, D. (2016). Spatio-temporal structure, path characteristics, and perceptual grouping in immediate serial spatial recall. Frontiers in Psychology, 7, 1686.

Frensch, P. A., \& Miner, C. S. (1994). Effects of presentation rate and individual differences in short-term memory capacity on an indirect measure of serial learning. Memory \& Cognition, 22(1), 95-110.

Graziano, M. S. A., Taylor, C. S. R., \& Moore, T. (2002). Complex movements evoked by microstimulation of precentral cortex. Neuron, 34(5), 841-851.

Haider, H., \& Frensch, P. A. (2009). Conflicts between expected and actually performed behavior lead to verbal report of incidentally acquired sequential knowledge. Psychological Research Psychologische Forschung, 73(6), 817-834.

Hawkins, H. L., MacKay, S. L., Holley, S. L., Friedin, B. D., $\&$ Cohen, S. L. (1973). Locus of the relative frequency effect in choice reaction time. Journal of Experimental Psychology, 101(1), 90-99.

Hebb, D. O. (1949). The organization of behavior: A neurophysiological theory. New York: Wiley.

Heitz, R. P. (2014). The speed-accuracy tradeoff: History, physiology, methodology, and behavior. Frontiers in Neuroscience, 8, 150.

Hikosaka, O., Miyashita, K., Miyachi, S., Sakai, K., \& Lu, X. (1998). Different roles of the frontal cortex, basal ganglia, and cerebellum in visuomotor sequences. Neurobiology of Learning and Memory, 70(1-2), 137-149.

Hikosaka, O., Nakahara, H., Rand, M. K., Sakai, K., Lu, X., Nakamura, K., ... Doya, K. (1999). Parallel neural networks for learning sequential procedures. Trends in Neuroscience, 22(10), 464-471.

Hikosaka, O., Nakamura, K., Sakai, K., \& Nakahara, H. (2002). Central mechanisms of motor skill learning. Current Opinion in Neurobiology, 12(2), 217-222.

Hommel, B. (1994). Spontaneous decay of response-code activation. Psychological Research, 56(4), 261-268.

Hommel, B. (2000). Intentional control of automatic stimulusresponse translation. In Y. Rossetti \& A. Revonsuo (Eds.), Beyond dissociation: Interaction between dissociated implicit and explicit processing (pp. 221-242). Amsterdam: John Benjamins.
Hunt, R. H., \& Aslin, R. N. (2001). Statistical learning in a serial reaction time task: Access to separable statistical cues by individual learners. Journal of Experimental Psychology: General, 130(4), 658-680.

Kandel, S., \& Perret, C. (2015). How do movements to produce letters become automatic during writing acquisition? Investigating the development of motor anticipation. International Journal of Behavioral Development, 39(2), 113-120.

Karni, A., Meyer, G., Jezzard, P., Adams, M. M., Turner, R., \& Ungerleider, L. G. (1995). Functional MRI evidence for adult motor cortex plasticity during motor skill learning. Nature, 377(6545), 155-158.

Keele, S. W., Ivry, R., Mayr, U., Hazeltine, E., \& Heuer, H. (2003). The cognitive and neural architecture of sequence representation. Psychological Review, 110(2), 316-339.

Kornblum, S., Hasbroucq, T., \& Osman, A. (1990). Dimensional overlap: Cognitive basis for stimulus-response compatibility - A model and a taxonomy. Psychological Review, 97(2), 253-270.

Logan, G. D. (1988). Toward an instance theory of automatization. Psychological Review, 95(4), 492-527.

Lowel, S., \& Singer, W. (1992). Selection of intrinsic horizontal connections in the visual cortex by correlated neuronal activity. Science, 255(5041), 209.

McLean, J. P., \& Shulman, G. L. (1978). On the construction and maintenance of expectancies. Quarterly Journal of Experimental Psychology, 30(3), 441-454.

Pashler, H., \& Baylis, G. (1991). Procedural learning: 1. Locus of practice effects in speeded choice tasks. Journal of Experimental Psychology: Learning, Memory, and Cognition, 17(1), 20-32.

Proctor, R. W., \& Vu, K.-P. L. (2013). Action selection. In A. F. Healy \& R. W. Proctor (Eds.), Handbook of Psychology. Experimental Psychology. (Vol. 4, pp. 295-319). Hoboken, New Jersey: John Wiley \& Sons.

Rosenbaum, D. A. (2017). Knowing Hands: The Cognitive Psychology of Manual Control. New York: Cambridge University Press.

Rosenbaum, D. A., Loukopoulos, L. D., Meulenbroek, R. G. J., Vaughan, J., \& Engelbrecht, S. E. (1995). Planning reaches by evaluating stored postures. Psychological Review, 102(1), 28-67.

Ruitenberg, M. F. L., Verwey, W. B., Schutter, D. J. L. G., \& Abrahamse, E. L. (2014). Cognitive and neural foundations of discrete sequence skill: A TMS study. Neuropsychologia, 56, 229-238.

Rünger, D., \& Frensch, P. A. (2008). How incidental sequence learning creates reportable knowledge: The role of unexpected events. Journal of Experimental Psychology: Learning, Memory, and Cognition, 34(5), 1011-1026.

Rünger, D., \& Frensch, P. A. (2010). Defining consciousness in the context of incidental sequence learning: theoretical considerations and empirical implications. Psychological Research Psychologische Forschung, 74(2), 121-137.

Shea, C. H., \& Kovacs, A. (2013). Complex movement sequences. How the sequence structure affects learning and transfer. In W. Arthur, E. A. Day, W. Bennett \& A. M. Portray (Eds.), Individual and team skill decay: The science and 
implications for practice (pp. 205-239). New York: Taylor/ Francis.

Shea, C. H., Kovacs, A. J., \& Panzer, S. (2011). The coding and inter-manual transfer of movement sequences. Frontiers in Psychology, 2, 1-10.

Shea, C. H., Panzer, S., \& Kennedy, D. (2016). Effector transfer. In F. Loffing, N. Hagemann, B. Strauss \& C. MacMahon (Eds.), Laterality in sports: Theories and applications (pp. 180-204). San Diego, CA: Academic Press.

Sternberg, S., Monsell, S., Knoll, R. L., \& Wright, C. E. (1978). The latency and duration of rapid movement sequences: comparisons of speech and typewriting. In G. E. Stelmach (Ed.), Information processing in motor control and learning (pp. 117-152). New York: Academic Press.

Theios, J. (1975). The components of response latency in simple human information processing tasks. In P. M. A. Posner \& S. Dornic (Eds.), Attention and performance $V$ (pp. 418-440). New York: Academic Press.

Verwey, W. B. (1995). A forthcoming key press can be selected while earlier ones are executed. Journal of Motor Behavior, 27(3), 275-284.

Verwey, W. B. (1996). Buffer loading and chunking in sequential keypressing. Journal of Experimental Psychology: Human Perception and Performance, 22(3), 544-562.

Verwey, W. B. (1999). Evidence for a multistage model of practice in a sequential movement task. Journal of Experimental Psychology-Human Perception and Performance, 25(6), 1693-1708.

Verwey, W. B. (2001). Concatenating familiar movement sequences: the versatile cognitive processor. Acta Psychologica, 106(1-2), 69-95.

Verwey, W. B. (2003). Processing modes and parallel processors in producing familiar keying sequences. Psychological Research, 67(2), 106-122.

Verwey, W. B., Abrahamse, E. L., \& De Kleine, E. (2010). Cognitive processing in new and practiced discrete keying sequences. Frontiers in Psychology, 1(32), 1-13.
Verwey, W. B., Abrahamse, E. L., De Kleine, E., \& Ruitenberg, M. F. L. (2014). Evidence for graded central processing resources in a sequential movement task Psychological Research, 78(1), 70-83.

Verwey, W. B., Abrahamse, E. L., Ruitenberg, M. F. L., Jiménez, L., \& De Kleine, E. (2011). Motor skill learning in the middle-aged: Limited development of motor chunks and explicit sequence knowledge. Psychological Research, 75(5), 406-422.

Verwey, W. B., \& Dronkers, W. J. (2018). Skill in discrete keying sequences is execution rate-specific. Psychological Research, 1-12.

Verwey, W. B., Groen, E. C., \& Wright, D. L. (2016). The stuff that motor chunks are made of: Spatial instead of motor representations? Experimental Brain Research, 234(2), 353-366.

Verwey, W. B., Shea, C. H., \& Wright, D. L. (2015). A cognitive framework for explaining serial processing and sequence execution strategies. Psychonomic Bulletin \& Review, 22(1), 54-77.

Verwey, W. B., \& Wright, D. L. (2004). Effector-independent and effector-dependent learning in the discrete sequence production task. Psychological Research, 68(1), 64-70.

Wickelgren, W. A. (1969). Context-sensitive coding, associative memory, and serial order in (speech) behavior. Psychological Review, 76, 1-15.

Wickelgren, W. A. (1977). Speed-accuracy tradeoff and information processing dynamics. Acta Psychologica, 41(1), 67-85.

Winer, B. J., Brown, D. R., \& Michels, K. M. (1991). Statistical principles in experimental design (3rd ed.). New York: McGraw-Hill.

Received February 16, 2018

Revised August 15, 2018

Accepted August 29, 2018 